\title{
Development of an Industrial Application with Neuro-Fuzzy Systems
}

\author{
Benaicha Sonia \\ Laboratory of Automation and Production \\ University of Batna-ALGERIA \\ 1, Street Chahid Boukhlouf 05000 Batna, Algeria \\ benaichas@yahoo.fr \\ Zermane Hannane \\ Laboratory of Automation and Production \\ University of Batna-ALGERIA \\ 1, Street Chahid Boukhlouf 05000 Batna, Algeria \\ hannanezermane@yahoo.fr
}

\author{
Mouss Hayet \\ Laboratory of Automation and Production \\ University of Batna-ALGERIA \\ 1, Street Chahid Boukhlouf 05000 Batna, Algeria \\ hayet_mouss@yahoo.fr \\ Bencherif Fateh \\ Laboratory of Automation and Production \\ University of Batna-ALGERIA \\ 1, Street Chahid Boukhlouf 05000 Batna, Algeria \\ f.bencherif@hotmail.fr
}

\begin{abstract}
In this paper, our objective is dedicated to the detection of a deterioration in the estimated operating time by giving preventive action before a failure, and the classification of breakdowns after failure by giving the action of the diagnosis and / or maintenance. For this reason, we propose a new Neuro-fuzzy assistance prognosis system based on pattern recognition called "NFPROG" (Neuro Fuzzy Prognosis). NFPROG is an interactive simulation software, developed within the Laboratory of Automation and Production (LAP) -University of Batna, Algeria. It is a four-layer fuzzy preceptor whose architecture is based on Elman neural networks. This system is applied to the cement manufacturing process (cooking process) to the cement manufacturing company of Ain-Touta-Batna, Algeria. And since this company has an installation and configuration S7-400 of Siemens PLC PCS7was chosen as a programming language platform for our system.
\end{abstract}

Keywords- Pattern Recognition ; Neuro-Fuzzy System ; Elman network ; PCS7

Much work has been dedicated to elaboration of assistance diagnosis systems. The majority of those systems are based on computer science tools by coupling methods of artificial intelligence such as fuzzy logic, expert systems and neural networks.

So the neuro-fuzzy systems are used to solve the problems of classifying the failures. But, when a supervision method estimates the future conditions of a system from its current state, it is called in this case, predictive diagnosis (prognosis). This acknowledgment of the past signal is only possible with the temporal architectures of neural networks. Infact, static neural networks are unable to ensure such processing. Early detection of any stage of degradation or even the learning of a sequence of evolution of a system requires a temporal neural architecture (dynamic).
The objective of this work is the development of a prognosis system is based on the recognition of the temporal neuro-fuzzy patterns, which rely on a digital representation and symbolic forms at the same time. The practical realization of our work is concerns the industrial field, specifically that cooking process of the cement manufacturing company of Ain-ToutaBatna-Algeria.

Taking into account the perspectives of work [4] and [5], which are applied to the workshop of cement cooking to resolve the problem of diagnosis, our study allows the supervising of behavior of cooking process, an esteem of the length of time taken by an operation before a failure and risk of later appearance of one or more failure modes, and the classification of breakdowns. This solution gives birth to a temporal neural industrial prognosis tool "NFPROG" (Neuro Fuzzy PROGnosis).

\section{PATTERN RECOGNITION TOOLS FOR INDUSTRIAL PROGNOSIS}

Pattern Recognition methods assume that no model is available to describe the relationship of cause to effect. The only knowledge based on human expertise supported by a solid feedback.

\section{A. The Fuzzy Logic}

Fuzzy logic was developed by Lotfi Zadeh (1965) to model human knowledge representation, and to improve the performance of systems that use this modeling decision. It is introduced in the assistance fields for the diagnosis in the decision making and in all knowledge-based systems. 


\section{B. Artificial Neural Network}

The Artificial Neural Networks (ANN) are a tool well suited to problems of perception, classification and prediction. Their use falls particularly in the supervising methodologies in the absence of process model. The use of ANNs is mainly guided by their properties as learning ability, the parallelism in the processing, the adaptation to nonlinear systems and processing speed.

\section{Elman Neural Network}

The dynamic (temporal) neural networks process time in a manner totally internal in the network; they have the ability to store information. The Elman network is one of the types of temporal networks. This network consists of returning the hidden layer to the input layer. Then there is a conservation of the trace of the internal activities of the network on a time step (Figure 2).

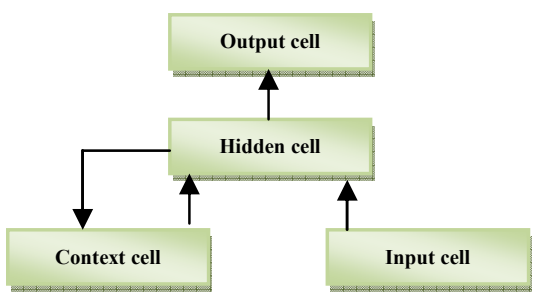

Figure 2: Elman Network

Each hidden cell is connected to a single context cell by a fixed connection. The internal state is defined by all the cells of the hidden layer.

\section{Neuro-Fuzzy Systems}

The neuro-fuzzy systems were born from the combination of neural networks and fuzzy logic, so as to take profits from the advantages of each one of these two techniques. The main property of neuro-fuzzy systems is their ability to process in the same tool numerical and symbolic knowledge of a system. They can therefore exploit the learning capabilities of neural networks in one hand and reasoning abilities of fuzzy logic on the other.

\section{COOKING WORKSHOP}

Our system will be exploited within the cooking workshop of the cement manufacturing company of Ain-Touta-BatnaAlgeria. Each one of the involved operations in the of the cement manufacturing process is important and must be correct; otherwise the cement may not have the quality required for its use. However, cooking is probably the most sensitive operation, the largest and during which essential chemical reactions will be make. It largely determines the quality of the cement.

The oven is a rotary steel cylinder installed with an inclination of 3 to $5 \%$ to the exit. It is calibrated at intervals by carrying roller trains. The rotation is transmitted through a ring gear, a pinion and a speed reducer. It is protected against very high operating temperatures by coating refractory brick masonry on the inside of any length. Raw materials are baked in the oven inlet (supply) and move against the current of hot gases (sintering) until they come out (cooler) in the form of clinker done (Figure 3).
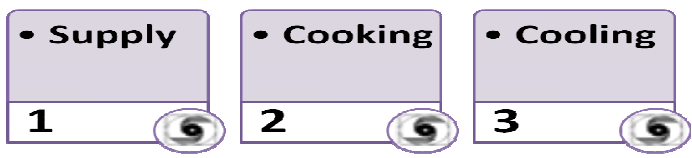

Figure 3: Different areas of the cooking workshop the oven

\section{WORK ENVIRONNEMENT}

The PCS 7 is the platform used for the implementation of our new system. Several tools are integrated into the PCS7 [6], which can be summarized in the following:

$>$ The S7 consists of a power supply, a CPU and an input or output module. The PLC monitors and controls a machine or process with the help of S7 program [6].

$>$ PCS7 is a process control system from Siemens who, with many automatic features. These are the basic objects present regardless of the number of operator stations, modules or their networking.

$>$ SIMATIC Manager, the core application and the gateway to all other applications used to create a PCS 7 project.

> S7-PLCSIM, the simulation software of automats for the development of programs without having the target PLC.

D 5 -GRAPH is program sequencers(GRAFCET).

$>$ WinCC (Windows Control Center) is a software for supervison and configuration in a single-or multifunctioning post. It is a graphics system for displaying and controlling the process views. So the interface is executed in the execution time of WinCC, where the operator can enter data, view information, start or stop the progress of the system.

$>$ FuzzyControl++ is a tool dedicated to the Siemens system configuration of fuzzy logic. It uses as a method of inference, the proven method TakagiSugeno. The on-line analysis of the Fuzzy logic systems is performed by using of curves and graphs tools, 2, 3 or 4 dimensions.

$>$ NeuroSystems supports not only learning, but also the configuration and the analysis of neural networks. It allows easy optimization of multilevel preceptors, radial basis networks and mixed neuro-fuzzy networks. On-line analysis systems of neural networks is done realized by using of curves and graphs tools 2, 3 or 4 dimensions.

\section{TOWARDS A NEURO-FUZZY OF PATTERN RECOGNITION: INDUSTRIAL APPLICATION}

In our developed approach, the function of the neuro-fuzzy system by Pattern Recognition for the prognosis pursuits three phases (figure 4): 


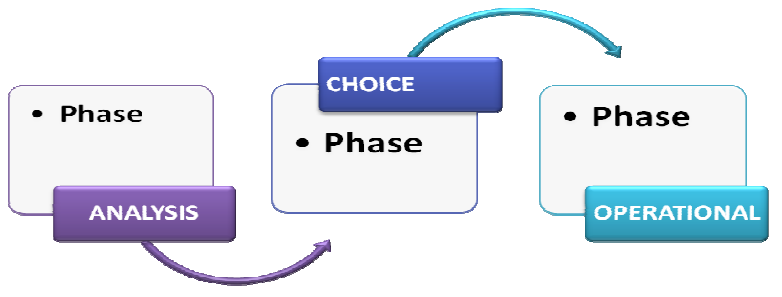

Figure 4: Different phases of the system by Pattern Recognition

\section{A. Analysis Phase}

The oven process is influenced by internal and external disturbances, and all these disturbances thwart the control. For this reason, we applied the method FMECA (Failure Modes, Effects and Criticality Analysis) in the oven to establish the connections of cause to effect between failed components and the observed symptoms. These links are represented in the form of fuzzy rules building the knowledge base. The architecture of our Neuro-Fuzzy System is illustrated in Figure 5; it is a recurrent neuro-fuzzy preceptor of 4 layers, 8 inputs and 4 outputs

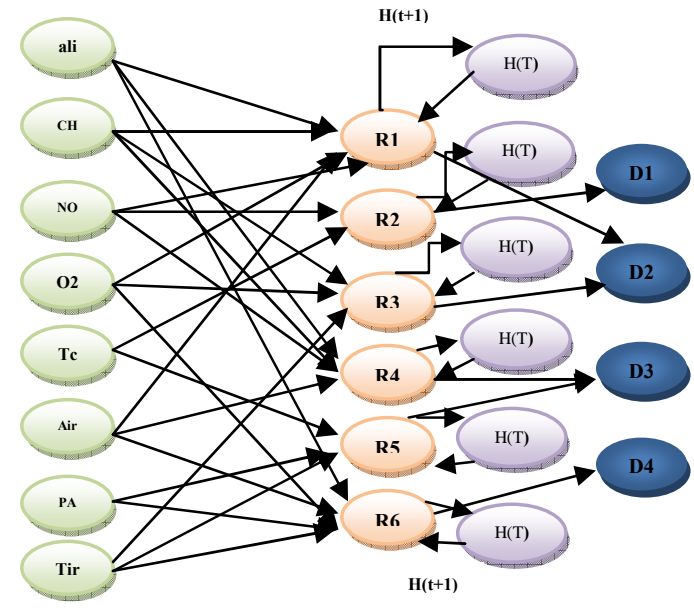

Figure 5: Neuro-Fuzzy Temporal System proposed for cooking workshop; Elaborated by the authors

The input layer is the set of numeric or symbolic forms observed in system, whose weights are modeled by fuzzy sets, the output layer is the set of failure modes of the studied system, and the hidden layer is a set of the fuzzy rules, a duplication of the hidden layer on a layer called context of weight equal to 1 .

\section{B. Choice Phase}

After giving the structure of our system, we need to associate each input variable a finite number of fuzzy sets and use the type of membership function associated, and also the interval of definition of variables and/or fuzzy sets.

NFPROG FuzzyControl ++ uses to give partitions of each input variable (Figure 6); whose the inference method is Sugeno (Sum-Min inference).

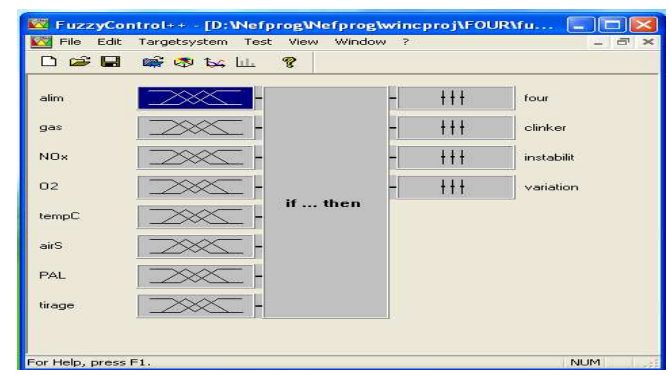

Figure 6: Graphical interface of the FuzzyControl ++

The membership functions are defined using the FuzzyControl + + tool (Figures 7, 8).

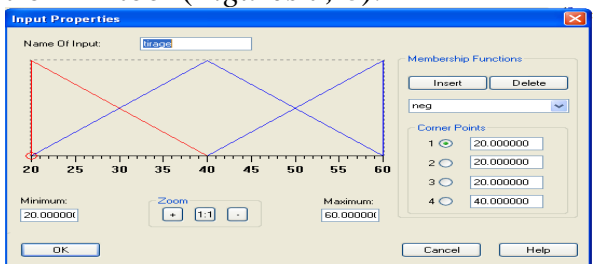

Figure 7: Input - drawing

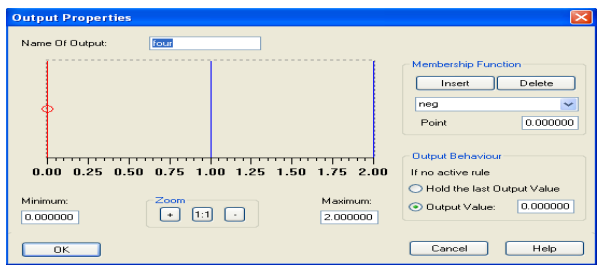

Figure 8 : Output - Oven

The rules can be captured with a tabular or matrix editor (Figure 9).

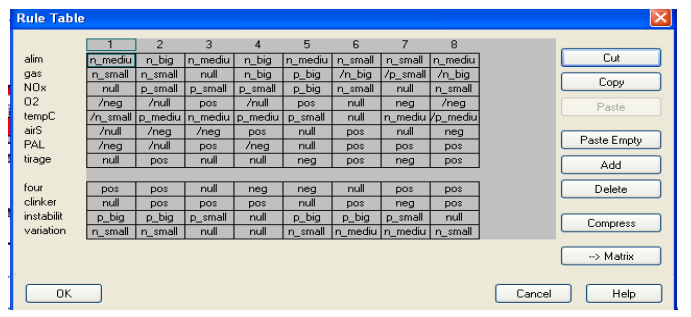

Figure 9: Table of Fuzzy Rules

The FuzzyControl++ tool generates the curve and the surface of the oven parameters (Figures 10, 11).

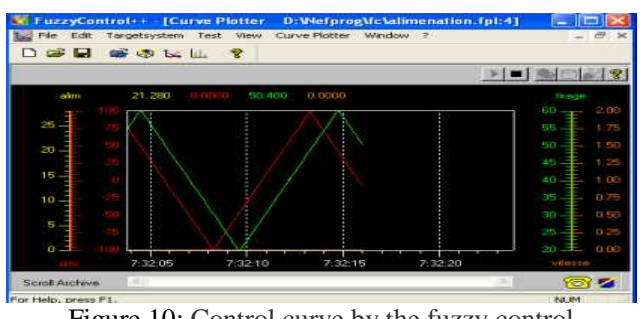

Figure 10: Control curve by the fuzzy control

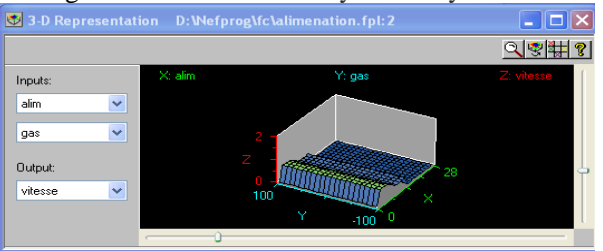

Figure 11: Surface generated by the fuzzy control 
After creating all the rules and fuzzy sets, a next step is the learning.

NeuroSystems allows the configuration of three types of neural networks; we choose the type "NFN" (Neuro Fuzzy Network). The Graphic interface of our system, via NeuroSystems, is illustrated in Figure 12.

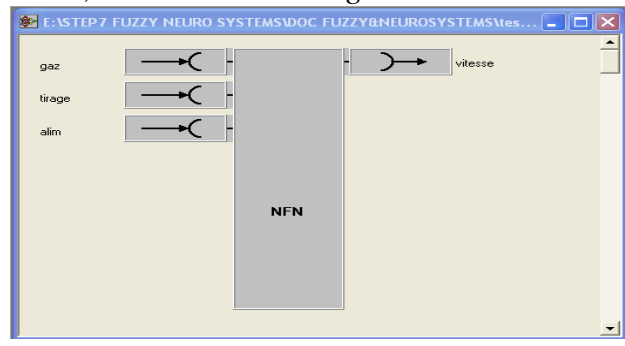

Figure 12: NeuroSystems Interface

The main steps of learning are presented as follows:

- Initialization: for each given outcome of the sensors, there is an input unit, and for each failure mode there is an output unit. For each input unit an initial fuzzy partition is specified.

- Learning the rules: the system can start and refine a basic knowledge of partial forms during learning. The rule will be created and added to the basic rules for research if not identical to the existing rules.

- Learning the membership functions: for learning membership functions, a simple back propagation is used. A decision will be created which depends on the error output for each unit rules. Each rule has changed its membership functions by changing their supports.

After the learning the curve and the surface can be generated by NeuroSystems (Figures 13, 14).

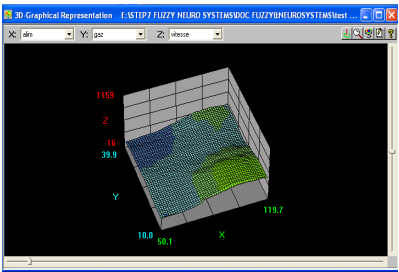

Figure 13: Surface generated

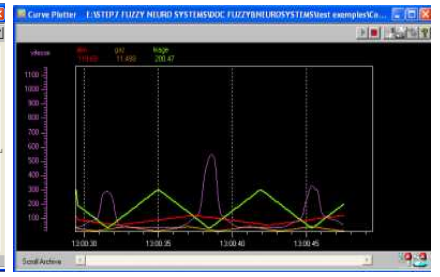

Figure 14: Control Curve
After the learning step, a test step occurred (Figure 15).

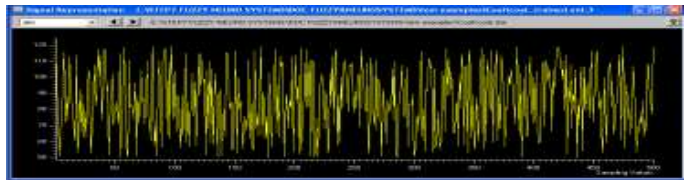

Figure 15: Test step

\section{Operational phase}

This phase is to implement the system to propose a decision for any further comments made. The following section explains the details of this phase in our system. After applying the three phases of the Pattern Recognition (Figure 16) on our approach, we obtain a new system called Neuro-Fuzzy Time "NFPROG".

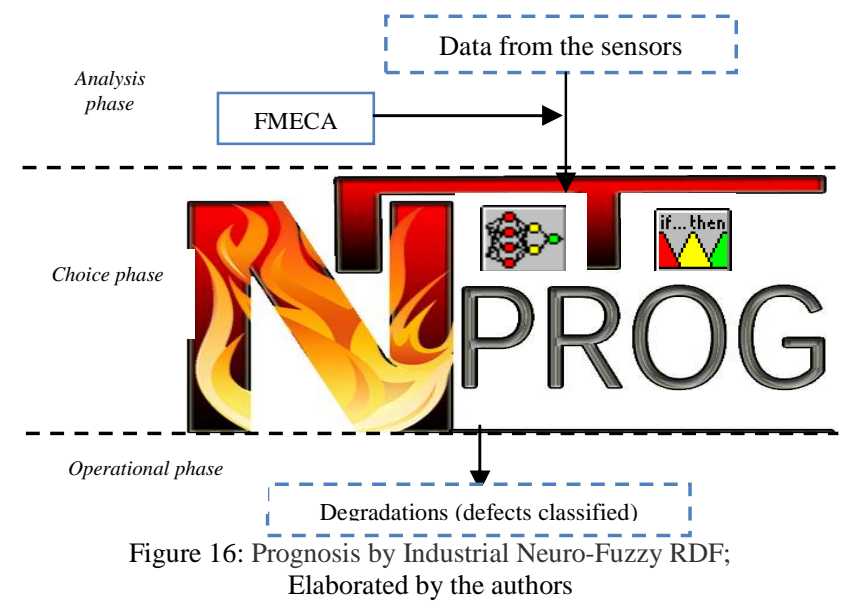

\section{Alarms and Pre-alarms}

The concept alarm is introduced into a diagnosis system while the prognosis system knows it as a pre-alarm (concept). The two concepts (Figure 17) allow the warning to the operator that there are failures.

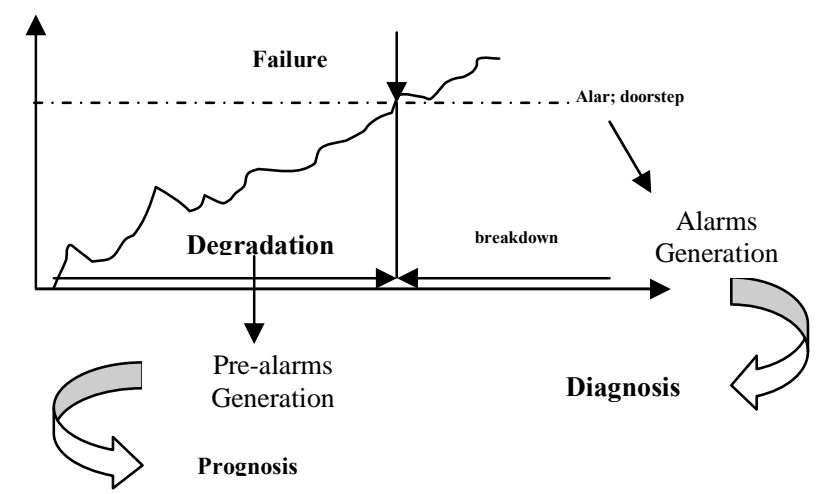

Figure 17: Pre-alarms and alarms Generation

\section{E. Interface NFPROG}

Our system consists of five views; the general interface of the cooking workshop is illustrated in Figure 18.

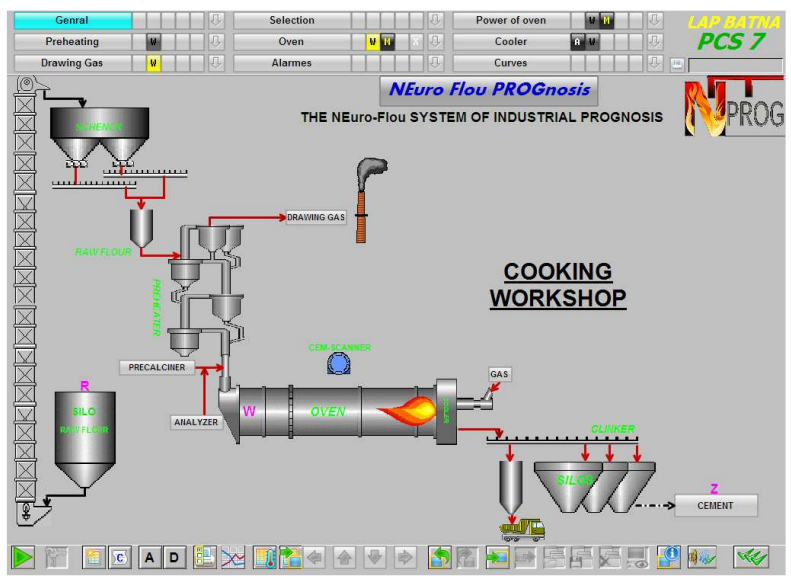

Figure 18: NFPROG General Interface

The view selection has allowed us the start selection of the workshop equipment (Figure 19). 


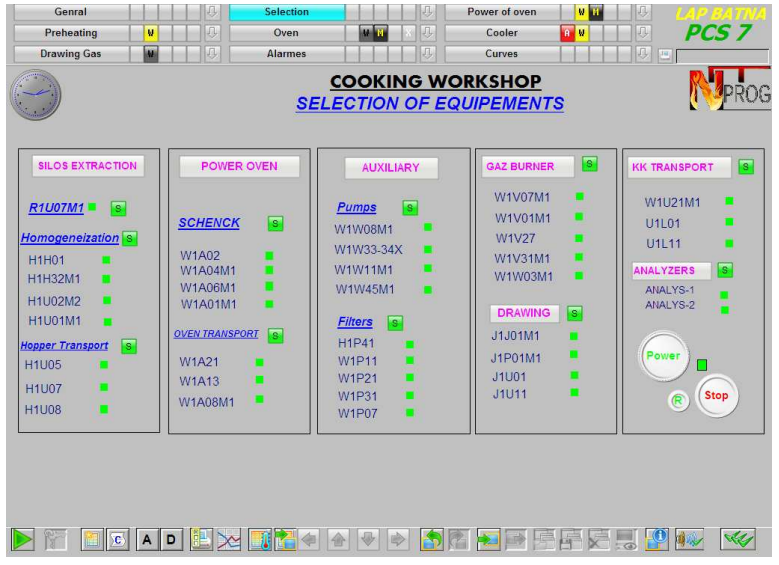

Figure 19: Selection view

The power supply of the oven (Figure 20) is synchronized by the raw material flow, drawing gas and the number of revolutions of the oven.

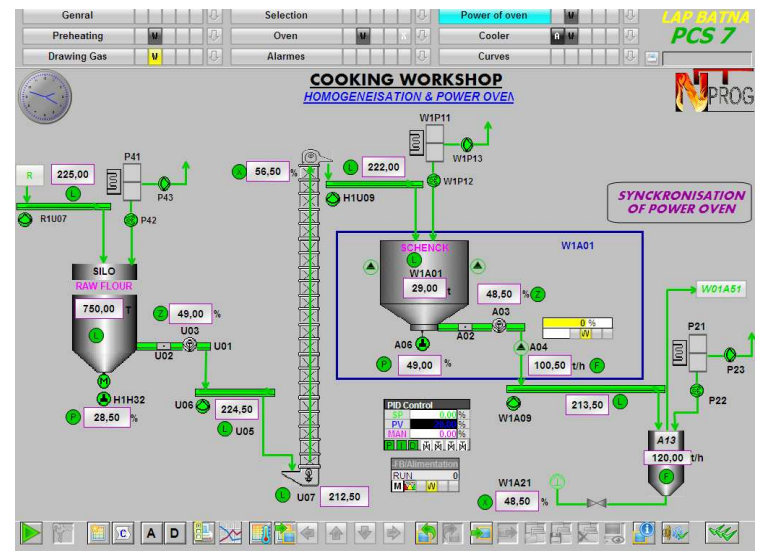

Figure 20: the power supply of the oven

The preheating step is an important step for heating the oven. The operator can view and change the various parameters of preheating (Figure 21).

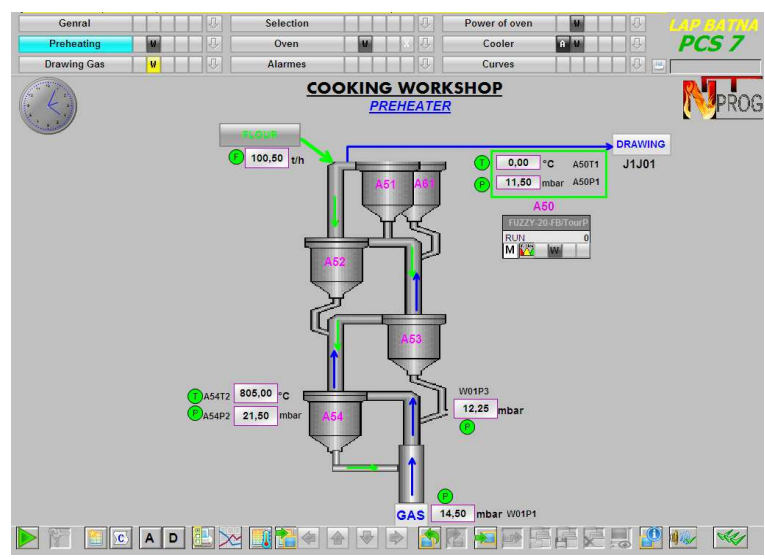

Figure 21: Preheating tower

The purpose of cooking is shown in Figure 22. Several parameters are shown; they are either entered manually or calculated from other parameters. This is the essential part of NFPROG.

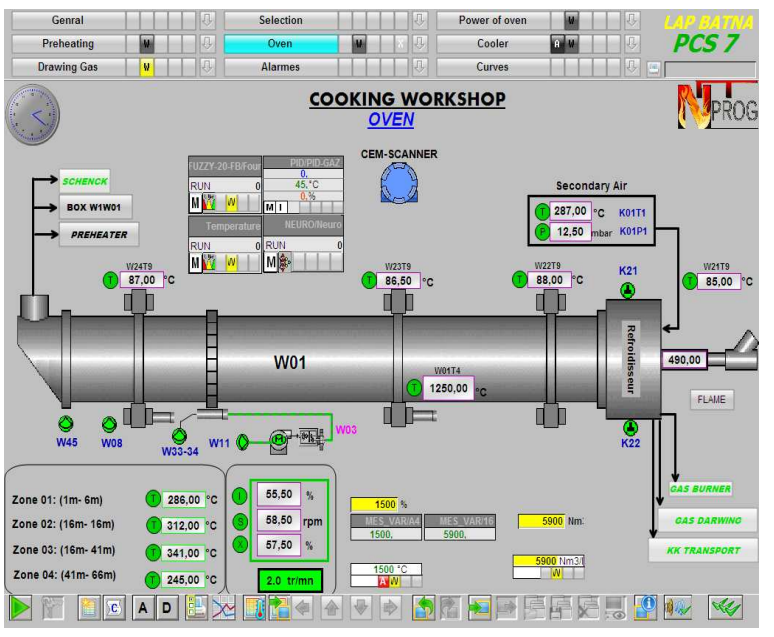

Figure 22: Cooking zone

The final stage of the sintering process is cooling, then the transport of Clinker (Figure 23).

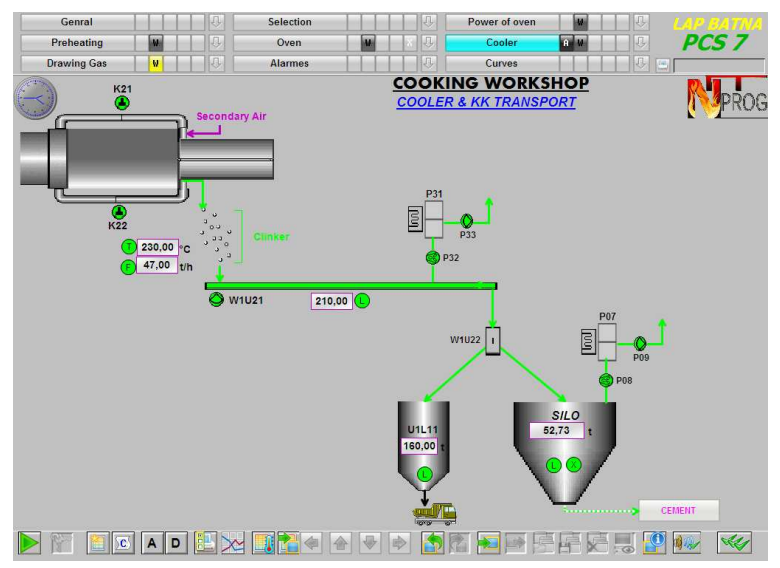

Figure 23: Clinker cooling and storage

The draw gas going in the opposite direction of the material. It can draw the gas and recover the raw material through a filter (figure24).

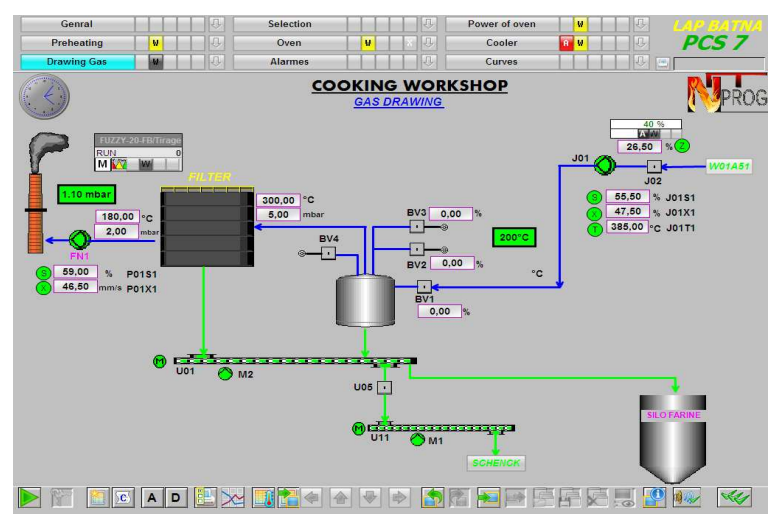

Figure 24: Draw gas

Alarms are displayed in the Alarms view. The parameters are controlled by regulators; the pre-alarms are displayed if there is one or more degradation of the system. After a warning to the operator by a pre-alarm, with a preventive action proposed to resolve the problem, the operator can intervene for a 
predictive diagnosis to eliminate this pre-alarm, otherwise, an alarm will be recorded and displayed (Figure 25) and in this case, a diagnosis is mandatory.

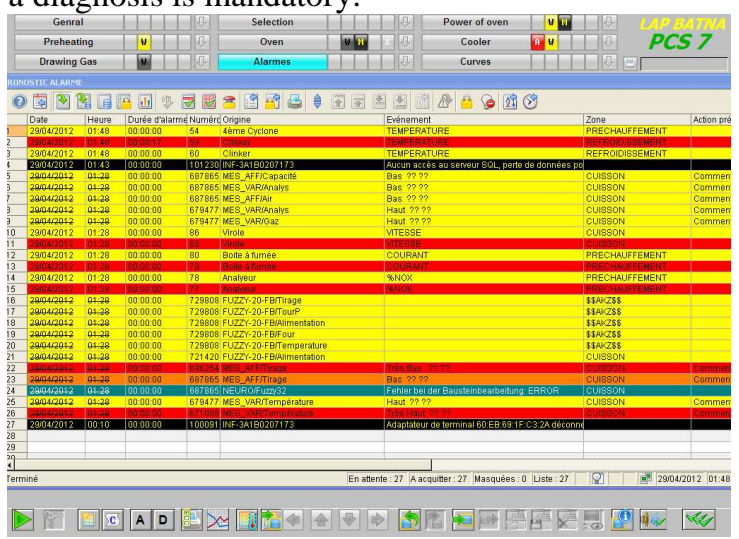

Figure 25: Alarm and Pre-Alarm View

The dynamic curves of the system are displayed in the view curves (Figure 26).

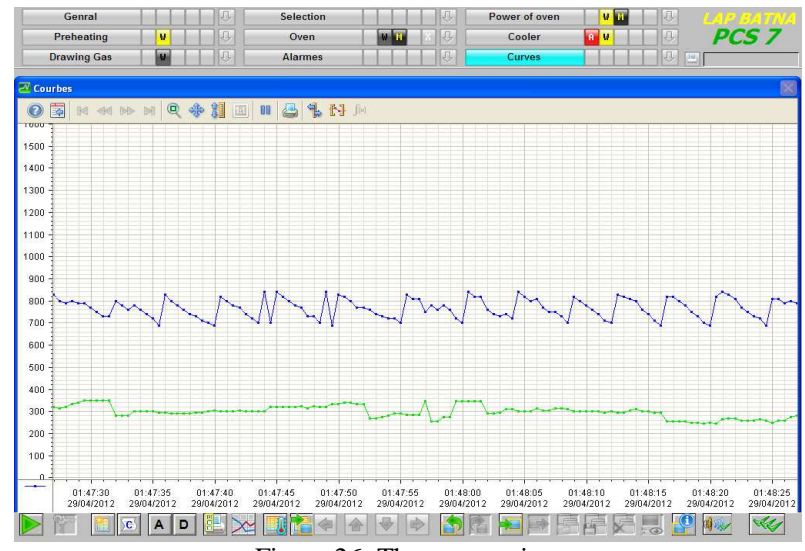

Figure 26: The curves view

\section{CONCLUSION}

The activity of prognosis is a key industrial maintenance strategy. It has the ability to detect and isolate imminent faults, as well as to predict the future state of a system. Therefore, the usefulness of a prognostic system is linked to predict the evolution of the degradation of equipment.

The work provided in this article seeks the specification of an application framework for the implementation of a prognosis system that can predict the behavior of a system taking into account the dynamics of real equipment.

In the case of industrial processes, a large amount of drawbacks and a decrease in production are usually the result of a failure in the process and the solution is to correct it in time.

For this reason, a new temporal Neuro-fuzzy approach for industrial prognosis is made: NFPROG. It permits to predict future failures or degradations of an equipment in real time within the LAP-Batna and applied to the cooking workshop because of elevated numbers of failures of the oven area than other areas.

\section{REFERENCES}

[1] Vasile, O.E. (2008): Contribution au pronostic de défaillances par réseau neuro-flou : maitrise de l'erreur de prédiction. France.

[2] Koujok, M. (2010) : Contribution au pronostic industriel : intégration de la confiance à un modèle prédictif. France.

[3] Amine,M. ; Kamal, M. (2008) : Diagnostic et pronostic de défaillances par réseaux bayésiens. Cedex, France.

[4] Rafik, M. (2008) : Diagnostic industriel par neuro-flou -application à un systeme de production. Batna,Algérie.

[5] Djamel, M. (2005) : Diagnostic et conduite des systèmes de production par approche à base de connaissances. Batna,Algérie.

[6] SIEMENS.SIMATIC (2006): Programmer avec STEP 7- Manuel 6ES7810-4CA08-8CW1". Allemagne.

[7] SIEMENS. SIMATIC.(2006) : Système de conduite de process PCS 7 Configuration PC et autorisation (V7.1)Manuel d'utilisation.Allemagne.

[8] SIEMENS-SIMATIC.(2009) : Système de conduite de processus PCS 7OS Conduite du process (V7.1)- Instructions de service.Allemagne.

[9] SIEMENS_SIMATIC. (2009) : Système de conduite de processus- PCS 7- SFC pour SIMATIC S7 Manuel de programmation et d'utilisation. Allemagne.

\section{Creative Commons Attribution License 4.0 (Attribution 4.0 International, CC BY 4.0)}

This article is published under the terms of the Creative Commons Attribution License 4.0

https://creativecommons.org/licenses/by/4.0/deed.en_US 